\title{
Densely Calcified Polytetrafluoroethylene Right Ventricular out flow tract patch explanted after 33 years
}

\author{
Sukhjeet Singh, BalramAiran, SachinTalwar*
}

Department of Cardiothoracic and Vascular surgery, All India Institute of Medical Sciences, New Delhi, India

"Corresponding author: SachinTalwar, Department of Cardiothoracic and Vascular surgery, All India Institute of Medical Sciences, New Delhi, India, Tel: +911126594835, Fax:+911126588663, Email: msachintalwar@hotmail.co.

Citation: Sachin T, Sukhjeet S, Balram A (2017) Densely Calcified Polytetrafluoroethylene Right Ventricular out flow tract patch explanted after 33 years. Cardiolog Res Cardiovasc Med 2: 113. DOI: 10.29011/2575-7083.000013

Received Date: 13 March, 2017; Accepted Date: 30 March, 2017; Published Date: 6 April, 2017.

\begin{abstract}
Right ventricular outflow tract reconstruction following intracardiac repair of tetralogy of Fallot often requires placement of a patch. The choice of the patch material is variable and depends upon surgeon preference, availability and costs of the patch material. Available patch materials for the purpose are autologous pericardium (fresh unfixed or fixed in glutaryldehyde), PolyTetraFluoroEthylene (PTFE), homograft patches, bovine pericardium and recently the patches fashioned out of porcine intestinal mucosa or patched of adapted collagen cardiovascular scaffold. Despite the claimed advantages of each of these, long-term concerns include calcification, aneurysmal dilatation and development of dense adhesions to the surrounding tissues that make a re-operation difficult and hazardous. The image presented in this report shows a densely-calcified PTFE patch that was explanted on re-operation, 33 years after intracardiac repair of TOF.
\end{abstract}

Keywords: Patch; Right Ventricular Outflow Tract Reconstruction; Tetralogy of Fallot

\section{Case Report}

A 45-year-old male who underwent intracardiac repair for a diagnosis of TOF 33 years ago. The patient had undergone intracardiac repair for Tetralogy of Fallot. At that time, the right ventricle was opened in its outflow and the incision was extended across the pulmonary valve annulus ventricle into the main pulmonary artery. The hypertrophied infundibular muscle was resected, the ventricular septal defect was closed and finally the right ventricular outflow tract was reconstructed using a PTFE patch sutured to the ventriculotomy and extending across the ventriculotomy into the main pulmonary artery.

At this admission, he had dyspnea on exertion NYHA Class III. Detailed evaluation demonstrated severe right ventricular dysfunction and pulmonary insufficiency without any obstruction to the right ventricular outflow. His electrocardiogram is shown in Figure 1 which demonstrates evidence of right ventricular enlargement (Figure 1). Preoperative contrast Magnetic Resonance Im- aging (MRI) show the calcified patch across the right ventricular outflow (Figure 2) and a subsequent coronary angiogram demonstrated normal coronaries and calcification in the area of the right ventricular outflow (Figure 3).

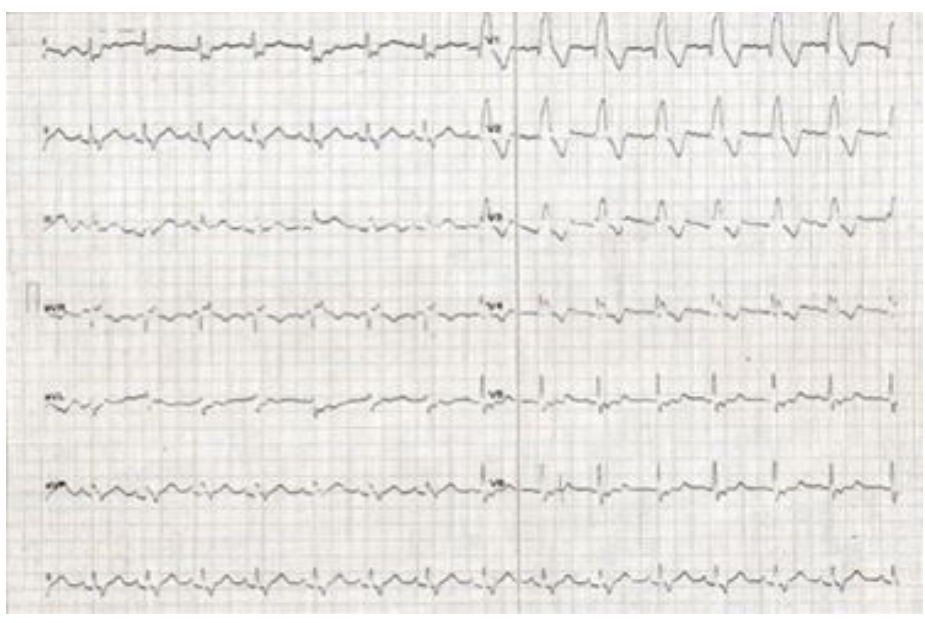

Figure 1: Pre-Operative Electrocardiogram Demonstrates Evidence of Right Ventricular Enlargement. 


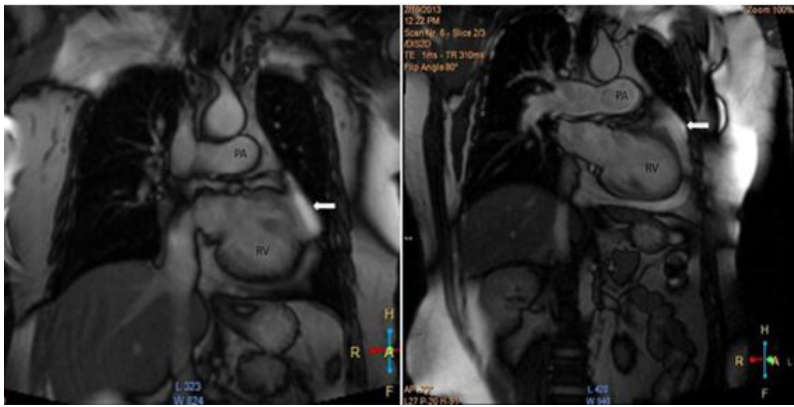

Figure 2: Preoperative Contrast Magnetic Resonance Imaging (MRI) Show the Calcified Patch across the Right Ventricular outflow (Arrow)

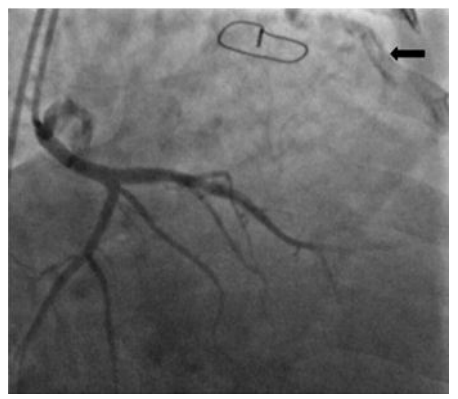

Figure 3: Angiogram Demonstrates Normal Left Coronary Arterial System and Calcification in the area of the Right Ventricular outflow (Arrow)

After obtaining informed consent, the patient underwent a re-operation via a median sternotomy approach. At surgery, dense adhesions were encountered. To our surprise, the PTFE patch was found to be densely calcified (Figures 1,2 ), bony hard on palpation and obscured the entire external cardiac anatomy. This patch was excised with great difficulty using heavy scissors. Following this, a bio prosthetic valve was implanted in the pulmonary position and the right ventricular outflow tract was reconstructed using a patch of bovine pericardium. Post-operative recovery was uneventful.

\section{Comments}

PTFE is the material of choice for the manufacture of synthetic vascular grafts and conduits, owing to its high flexibility, porosity, and electronegative surface charge that reduce the thrombogenic potential. PTFE is also said to resist natural deterioration in situ[1]. However, there are no long-term data on the fate of indwelling PTFE patches and grafts. With improving survival and increasing duration of follow-up of patients who have undergone intracardiac repairs in an earlier era, re-operations are increasing in these patients and offer us insights into the fate of these patch materials at long-term follow-up. In this case, it was as long as 33 years. Amongst the commonly used patch materials, calcification of PTFE is now considered an important problem that affects its function and durability. In vitro studies have demonstrated that PTFE graft has a greater potential for calcification than polyurethane, silicone and dacron[2,3]. In 2007, Hayabuchi et al[4], evalu- ated the radiological presence of calcification within PTFE vascular grafts by Multidetector-Row Computed Tomography (MDCT) imaging. In this review of 76 patients, calcification was detected in 26 of $32(81 \%)$ patients receiving a PTFE patch.

Previous studies on histo-pathological analysis of explanted PTFE membranes and conduits in another study have also revealed variable degrees and patterns of interstitial calcification, in addition to neointimal hyperplasia, foreign body reaction, and thrombosis without any association with patient's age, serum calcium, creatinine level, and blood urea nitrogen levels [5]. It is speculated that, the porosity of PTFE encourages formation of a neo-intimal lining, that facilitates the permeation of calcium and phosphorus ions, yielding calcium deposits within the interstices of grafts. This can act as a nidus for thrombus formation, can result in endothelial dysfunction and eventually graft failure/fracture[5].However, in our patient, other than the calcification, the PTFE graft otherwise functioned well for 33 years without thrombosis, fracture or aneurysmal dilatation. To the best of our knowledge, the present case represents the longest reported indwelling PTFE patch in the right ventricular outflow that was severely calcified. The supplied images elegantly demonstrate this degree of calcification, which was a surgical challenge that was managed appropriately (Figure 4).

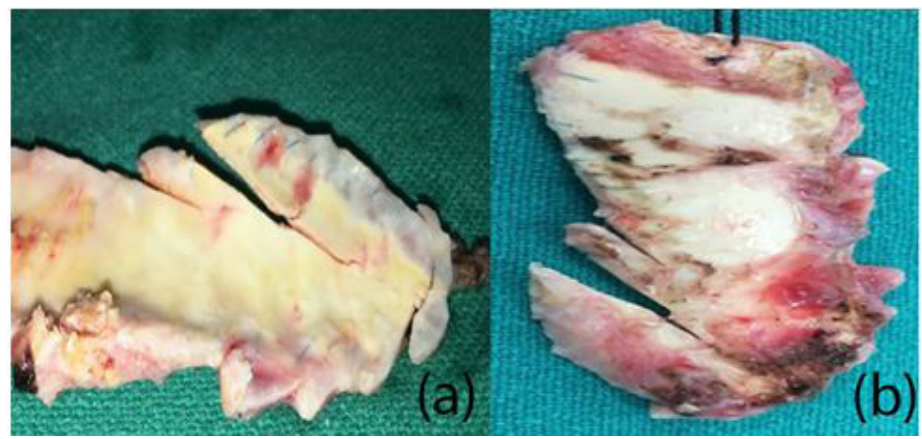

Figure 4: (a) and (b) Represent the Calcified Polytetrafluoroethylene Patch. The Patch was found to be Densely Calcified, Bony Hard on Palpation and Obliterated the Original Anatomy.

\section{References}

1. Hill D (1998) Design Engineering for Biomaterials and Medical Devices. Storrington, UK: John Wiley \& Sons Ltd 54-92.

2. Randall RD, Walley BD, Meredith JH (1982) Comparison of Poly Tetra Fluoro Ethylene (PTFE) and dacron as long, small-diameter arterial grafts in dogs. Am Surg 48: 622-627.

3. Park JC, Song MJ, Hwang YS, Suh H (2001) Calcification comparison of polymers for vascular graft. Yonsei Med J 42: 304-310.

4. Hayabuchi Y, Mori K, Kitagawa T, Sakata M, Kagami S (2007) Polytetrafluoroethylene graft calcification in patients with surgically repaired congenital heart disease: evaluation using multidetector-row computed tomography. Am Heart J 153: 806.e1-8.

5. Mehta RI, Mukherjee AK, Patterson TD, Fishbein MC (2011) Pathology of explanted polytetrafluoroethylene vascular grafts. Cardiovasc Pathol 20: 213-221. 